Bundesgesundheitsbl 2017 $\cdot 60: 707-714$

DOI 10.1007/s00103-017-2565-2

Online publiziert: 24. Mai 2017

(c) Der/die Autor(en) 2017. Dieser Artikel ist eine Open-Access-Publikation.

CrossMark
Karla Pfaff · Detlef Wölfle · Andreas Luch

Bundesinstitut für Risikobewertung, Berlin, Deutschland

\section{Kontaminanten aus Lebensmittelverpackungen}

\section{Neue Entwicklungen in der Risikobewertung}

\section{Einleitung}

In einem aktuellen Bericht konstatiert das Europäische Parlament eine Unterschätzung der Rolle von Lebensmittelkontaktmaterialien als Kontaminationsquelle für Lebensmittel [1]. Während generelle Anforderungen an Stoffe aus Materialien für den Lebensmittelkontakt auf europäischer Ebene festgelegt sind (Verordnung (EG) 1935/2004), gibt es außer für Kunststoffe (Verordnung (EU) 10/2011) nur wenige Regelungen für andere Materialien [2]. Es wird im Bericht des Europäischen Parlaments darauf hingewiesen, dass zwischen der Regulation von Materialien für den Lebensmittelkontakt und der EU-Chemikalienverordnung REACH eine engere Abstimmung insbesondere im Hinblick auf die als CMR-Stoffe (kanzerogen, mutagen, reproduktionstoxisch) oderSVHCs (substances of very high concern) eingestuften Substanzen erfolgen sollte. Geueke et al. [3] führen eine Reihe von CMR-Substanzen auf, die als Kunststoffmonomere und -additive (EU-Richtlinie 10/2011) oder als Stoffe in anderen Materialien [4] für den Kontakt mit Lebensmitteln gelistet sind. Entsprechend eingestufte oder als problematisch erkannte Substanzen (Beispiele in • Tab. 1) sind aufgrund von Risikobewertungen auf europäischer Ebene (Verordnung (EU) 10/2011) oder auf nationaler Ebene (z. B. Empfehlungen des Bundesinstituts für Risikobewertung, BfR) [5] mit Restriktionen für Anwendungen im Kontakt mit Lebensmitteln versehen worden. Vom Europäischen Parlament ist die mangelnde gegenseitige Anerkennung von nationalen Regularien kritisiert worden.
Einige Substanzen für den Lebensmittelkontakt sind in der Europäischen Union bisher noch nicht geregelt. Van Bossuyt et al. [17] schätzen, dass mehr als 6000 Einzelverbindungen in bedruckten Papieren und Kartonagen vorkommen, wobei die Mehrheit (77\%) nicht toxikologisch bewertet ist und viele davon oral aufgenommen werden können. Als weiteres Kontaminantenproblem ist die Exposition des Verbrauchers gegenüber Gemischen von (teilweise bewerteten) Einzelsubstanzen wie Phthalaten [18] oder Perfluorverbindungen [19] sowie komplexen Gemischen aus nicht völlig identifizierten Komponenten wie Mineralöl-Kohlenwasserstoffen [20] oder dem „forest of peaks“ aus Beschichtungen [21] zu nennen. Einen wesentlichen Anteil an den migrierenden Stoffen können sogenannte nicht beabsichtigt zugesetzte Substanzen (non-intentionally added substances, NIASs) wie Verunreinigungen, Reaktionsprodukte oder Oligomere haben, die bei der Substanzbewertung durch die Europäische Lebensmittelsicherheitsbehörde (EFSA) und die nationalen Behörden (z. B. BfR) eine immer größere Rolle spielen.

In diesem Beitrag sollen neben neuen Bewertungsansätzen [22] auch Risikoaspekte neuer Technologien angesprochen werden, wie der Verwendung von Nanomaterialien, aktiver und intelligenter Verpackungen und des Recyclingverfahrens im Bereich der Materialien für den Lebensmittelkontakt.

\section{Risikobewertung}

Die Aufnahme neuer Stoffe in die Positivlisten für Substanzen, die zur Her- stellung von Materialien für den Lebensmittelkontakt verwendet werden dürfen, erfolgt auf Antrag des Herstellers und im Ergebnis einer Risikobewertung. Sowohl bei Anträgen für europäische als auch für nationale Regelungen ist der EFSA Note for Guidance [23] zu befolgen (Bewertungskriterien s. - Infobox 1). Danach sind gegenwärtig Art und Umfang der toxikologischen Daten, die im Antragsverfahren vorgelegt werden müssen, abhängig von der Höhe des unter ungünstigsten vorhersehbaren Verwendungsbedingungen stattfindenden Übergangs des betreffenden Stoffes auf Lebensmittel [2].

\section{Neue Bewertungsansätze zur Exposition}

Bis heute wird bei der Risikobewertung von Stoffen aus Materialien für den Lebensmittelkontakt zum Vergleich mit toxikologisch abgeleiteten Grenzwerten von folgenden vereinfachenden Annahmen für die Expositionsabschätzung ausgegangen:

- Eine Person mit einem Körpergewicht von $60 \mathrm{~kg}$ verzehrt täglich $1 \mathrm{~kg}$ eines Lebensmittels, in das der Stoff in der höchsten vorhersehbaren Menge übergegangen ist.

- $1 \mathrm{~kg}$ Lebensmittel kommen in Kontakt mit $6 \mathrm{dm}^{2}$ der Oberfläche des Materials („EU-Verpackungswürfel“ mit einer Kantenlänge von $1 \mathrm{dm}$ ).

Eine wissenschaftliche Begründung für diese Annahmen ist nicht nachvollziehbar; es ist davon auszugehen, dass sie in Ermangelung von Informationen sowohl zu Verzehrsmengen als auch zur Verwendung von Materialien im Lebensmittel- 
Tab. 1 Beispiele für problematische Substanzen im Kontakt mit Lebensmitteln

\begin{tabular}{|c|c|c|}
\hline Substanzen & Lebensmittelkontaktmaterial & Problem \\
\hline Primäre aromatische Amine & $\begin{array}{l}\text { Druckfarben, Polyamid-Mate- } \\
\text { rialien }\end{array}$ & Kanzerogen $[6,7]$ \\
\hline Phthalate & PVC, Papier & Reproduktionstoxisch $[8,9]$ \\
\hline Perfluorverbindungen & Papier, Beschichtungen & Persistent $[10,11]$ \\
\hline Bisphenol A & Polycarbonate, Epoxy-Harze & $\begin{array}{l}\text { Endokrin aktiv, reproduktions- } \\
\text { toxisch }[12,13]\end{array}$ \\
\hline Aluminium & Aluminiumfolie, Assietten & Vielseitige Exposition [14] \\
\hline Biozide (Isothiazolinone) & Papier & $\begin{array}{l}\text { Reproduktionstoxisch, immu- } \\
\text { notoxisch, Resistenzentwick- } \\
\text { lung [15] }\end{array}$ \\
\hline Mineralöle (MOSH, MOAH) & Papier & Gemische, Datenlücken [16] \\
\hline
\end{tabular}

kontakt vereinbart worden sind. Dieses Szenario kann sowohl zu einer Überschätzung als auch zu einer Unterschätzung der Exposition führen, abhängig von der tatsächlichen Kontaktfläche (z. B. der Verpackungsgröße), den Verzehrsmengen und der zu betrachtenden Bevölkerungsgruppe (z. B. Erwachsene oder Kleinkinder).

Zur Verbesserung der Datenlage hinsichtlich der Verzehrsmengen einschließlich des Anteils an verpackten Lebensmitteln für verschiedene Altersgruppen und des Oberflächen-VolumenVerhältnisses bei verpackten Lebensmitteln $[24,25]$ entwickelte die EFSA neue Szenarien für die Expositionsabschätzung. Dabei sollte insbesondere geprüft werden, ob die gegenwärtige Annahme im Hinblick auf die auf das Körpergewicht (KG) bezogene Aufnahme von Lebensmitteln, d. h. $17 \mathrm{~g} / \mathrm{kgKG}$, ein ausreichendes Schutzniveau für Säuglinge und Kleinkinder gewährleistet und ob diese Annahme zu konservativ für Stoffe ist, die nur geringfügig Anwendung in Materialien für den Lebensmittelkontakt finden. Weiterhin sollte die Entwicklung hin zur Verwendung kleiner Verpackungen mit einem erhöhten OberflächenVolumen-Verhältnis berücksichtigt werden.

Es wurden vier verschiedene Verwendungskategorien für Materialien für den Lebensmittelkontakt gebildet, die dafür von der EFSA abgeleiteten Verzehrmengen sind in $\bullet$ Tab. 2 zusammengefasst.

Für die Verwendungskategorie 1 ergibt sich somit im Vergleich zu den bisher zugrunde gelegten Annahmen eine ca. 9fach höhere Exposition.

\section{Neue Bewertungsansätze zur Toxikologie}

Die toxikologischen Datenanforderungen für eine Substanzbewertung beruhen gegenwärtig auf einem abgestuften Verfahren entsprechend der Höhe der unter ungünstigsten Verwendungsbedingungen zu erwartenden Substanzübergänge auf Lebensmittel bzw. Lebensmittelsimulanzien (Testmedium, das Lebensmittel nachahmt) [23] unter Berücksichtigung von Sicherheitsaspekten (Verbraucherschutz), ethischen Erwägungen (Tierschutz) und der Praktikabilität der Prüfungen (humane und finanzielle Ressourcen). Die danach für die gesundheitliche Bewertung von Substanzen zur Herstellung von Materialien für den Lebensmittelkontakt geltenden Datenanforderungen sind in $\mathbf{0}$ Tab. 3 zusammen mit den Vorschlägen der EFSA zu neuen Bewertungsansätzen dargestellt.

Die abgestuften Anforderungen sind in das neuen Diskussionspapier der EFSA [22] aufgenommen worden: Die Migrationsstufen $(\mathrm{mg} / \mathrm{kg}$ Lebensmittel bzw. Lebensmittelsimulans) sind darin durch Expositionsstufen ( $\mu \mathrm{g}$ aufgenommene Substanz/kgKG pro Tag) ersetzt worden. Die Höhe der unteren Expositionsstufen orientiert sich am Konzept des "Threshold of Toxicological Concern" (TTC). Mit diesem Ansatz können Substanzen aufgrund von chemischen Strukturmerkmalen in Toxizitätsklassen („Cramer-Klassen“) eingeteilt und ihnen Expositionsschwellen zugeordnet werden, unterhalb derer eine gesundheitliche Beeinträchtigung unwahrscheinlich ist. Entsprechend wurde für die untere
Expositionsstufe für Lebensmittelkontaktmaterialien, in der im Wesentlichen nur Genotoxizitätsdaten und öffentlich zugängliche Informationen/Daten gefordert werden, ein Wert von $1,5 \mu \mathrm{g} / \mathrm{kgKG}$ pro Tag vorgeschlagen (Cramer-Klasse III) [22]. Zusätzliche Informationen sind in dieser Stufe dann erforderlich, wenn sich aufgrund der chemischen Struktur Hinweise auf mögliche toxische Wirkungen unterhalb der Schwellenwerte ergeben, z. B. Neurotoxizität (Organophosphate), endokrine Aktivität (Bisphenole), oder bei einer Akkumulation im Körper (Perfluorverbindungen wie PFOA) sowie grundsätzlich für Nanomaterialien. Für Substanzen, die aufgrund ihrer Struktur und ihres Metabolismus toxikologisch als wenig bedenklich gelten (Cramer-Klasse I), wurden diese Informationen auch für eine Exposition von bis $\mathrm{zu} 30 \mu \mathrm{g} / \mathrm{kgKG}$ pro Tag für ausreichend gehalten. Liegen die Expositionen gegenüber der fraglichen Substanz oberhalb der genannten Grenzen ( 1,5 bzw. $30 \mu \mathrm{g} / \mathrm{kgKG}$ pro Tag), sind zusätzliche Daten zur subchronischen Toxizität (90-Tage-Test im Versuchstier) und der Nachweis für ein Fehlen von Akkumulation im menschlichen Körper erforderlich. Bei Expositionen oberhalb von $80 \mu \mathrm{g} / \mathrm{kgKG}$ pro Tag sind auch die chronische Toxizität, die Kanzerogenität sowie die Reproduktionsund Entwicklungstoxizität zusammen mit toxikokinetischen Parametern $\mathrm{zu}$ prüfen (•Tab.3).

Grundsätzlich sollte jede Teststrategie für eine Substanz auf den bereits vorhandenen Informationen beruhen, d. h. entsprechende toxikologische Daten sollten in Datenbanken (z. B. der ECHA) oder in der frei zugänglichen wissenschaftlichen Literatur recherchiert werden. Hieraus könnten sich einerseits bei Hinweisen auf spezielle toxische Eigenschaften der Substanz weitergehende Datenanforderungen ergeben oder anderseits auch die Möglichkeit, auf bestimmte Studien, insbesondere Tierversuche, zu verzichten, wenn sich dies durch entsprechende Daten zu der Substanz selber oder zu einer toxikologisch sehr ähnlichen Substanz („read across“) wissenschaftlich rechtfertigen lässt. Ein solches Vorgehen stimmt mit dem Ziel der Europäischen Kommis- 
Bundesgesundheitsbl 2017 -60:707-714 DOI 10.1007/s00103-017-2565-2

(c) Der/die Autor(en) 2017. Dieser Artikel ist eine Open-Access-Publikation.

K. Pfaff · D. Wölfle · A. Luch

\section{Kontaminanten aus Lebensmittelverpackungen. Neue Entwicklungen in der Risikobewertung}

\section{Zusammenfassung}

Materialien, die in Kontakt mit Lebensmitteln kommen, sind sehr vielfältig und stellen eine wichtige Kontaminationsquelle für Lebensmittel dar. Die Risikobewertung für den Übergang von Substanzen aus den meisten Materialien ist mit Ausnahme von Kunststoffen und Zellglas in Europa zurzeit noch nicht einheitlich geregelt. Die Europäische Lebensmittelsicherheitsbehörde EFSA hat 2016 ein Gutachten zu neuen Entwicklungen in der Risikobewertung von Substanzübergängen in Lebensmittel zur Diskussion gestellt. Neben der Bewertung von Monomeren und Additiven können auch Übergänge von nicht beabsichtigt zugesetzten Substanzen (NIASs) wie Verunreinigungen, Zerfallsprodukten oder Oligomeren für die Risikobetrachtung relevant sein. Aktuelle Informationen zum Lebensmittelverzehr und zum Oberflächen-Volumen-Verhältnis bei Lebensmittelverpackungen bieten zusammen mit Migrationsstudien die Möglichkeit einer besseren Expositionsabschätzung bei Verbrauchern. Für viele Substanzen aus Verpackungsmaterialien und anderen Produkten sind die Übergänge in Lebensmittel gering. Bei Migrationen bis $50 \mathrm{ppb}$ ist nur die Prüfung auf ein genotoxisches Potenzial der Substanzen vorgesehen. Bei höheren Substanzübergängen sind tierexperimentelle Daten erforderlich. Um Tierstudien so weit wie möglich zu reduzieren, können neben der Auswertung bereits vorhandener Informationen zu toxikologischen Eigenschaften gegebenenfalls auch toxikologische Daten von chemisch ähnlichen Stoffen auf der Grundlage von Struktur- und Wirkungsbetrachtungen für die Substanzbewertung nutzbar gemacht werden. Für die Bewertung von NIAS ist es möglich, mithilfe von computergestützter Simulation („In-silico"-Methoden) das gesundheitliche Risiko abzuschätzen. Herausforderungen für die Risikobewertung ergeben sich durch neue Technologien wie die Verwendung von Nanomaterialien, aktiven und intelligenten Verpackungen sowie von recyclierten Kunststoffen, für die von der EFSA Risikobewertungen vor der Markteinführung von der EU-Kommission vorgeschrieben sind.

Schlüsselwörter

Materialien für den Kontakt mit Lebensmitteln · Expositionsszenarien · Nicht beabsichtigt zugesetzte Substanzen (NIASs) · In-silicoMethoden zur Risikobewertung · Neue Technologien

\section{Contaminants from food packaging. New developments in risk assessment}

\section{Abstract}

Diverse materials intended for contact with food are important sources of food contamination. Harmonised European regulations including whitelists (so-called "positive lists") of substances along with migration limits and restrictions exist for plastics and regenerated cellulose films only. The European Food Safety Authority (EFSA) is responsible for the risk assessment of substances prior to their authorization and inclusion into the positive lists. In 2016 the EFSA issued an opinion on recent developments in the risk assessment of substances migrating into food for public consideration. Also migration related to non-intentionally added substances
(NIASs), e. g. impurities, degradations products or oligomers, may be relevant for risk assessment. For substances migrating in quantities up to $50 \mathrm{ppb}$ the requested data are restricted to genotoxicity testing based on a tiered approach for toxicological data requirements. In the case of higher migration levels ( $>50 \mathrm{ppb}$ ) experimental animal studies are also requested. Along with an evaluation of the available information, toxicological data on structurally similar substances may be used for the assessment if sufficiently justified with the aim to reduce animal studies as far as possible. For the risk assessment of NIASs it is possible to apply in silico methods in the absence of experimental toxicological data. Additionally, new technologies such as the use of nanomaterials, active and intelligent packaging and recycled plastics are challenging tasks in EFSA's risk assessment in accordance with the regulations by the European Commission.

\section{Keywords}

Materials in contact with food - Exposure scenarios - Non-intentionally added substances (NIASs) - In silico methods for risk assessment . New technologies sion überein, unnötige Tierversuche zu vermeiden (Richtlinie 2010/63/EU; Artikel 4). Neuere toxikologische Untersuchungsstrategien einschließlich In-silico-Methoden sind im aktuellen EFSADiskussionspapier zu Lebensmittelkontaktmaterialien zur Diskussion gestellt worden [22].

Die EFSA hat ein Gutachten zu Teststrategien zur Evaluation des genotoxischen Potenzials einer Substanz im Rahmen der Risikobewertung veröffentlicht [26]. Als Basisanforderung werden darin zwei In-vitro-Testverfahren, der bakteri- elle Mutagenitätstest (OECD TG 471) zusammen mit einem In-vitro-Mikrokerntest (OECD TG 487) genannt, um die drei wichtigen genetischen Endpunkte zu erfassen: nämlich die mutagene Wirkungen und die strukturellen und numerischen Chromosomenschädigungen. Zur weiteren Genotoxizitätsprüfung einer Substanz, insbesondere bei positiven Testergebnissen in vitro, werden von der EFSA als geeignete In-vivo-Testverfahren der Mikrokerntest (OECD TG 474), der transgene Nager-Mutationstest (OECD TG 488) und der Comet-Assay
(OECD TG 489) vorgeschlagen. Diese Prüfstrategien werden auch für Substanzen mit Lebensmittelkontakt als geeignet betrachtet [22, 27]. Bei den vorgeschlagenen In-vivo-Testverfahren erlaubt die kombinierte Durchführung des Mikrokerntests zur Klärung von Chromosomenschädigung und des Comet-Assays als Indikatortest für primäre DNA-Läsionen (besonders an Organen wie Magen, Darm und Leber, die als erstes in Kontakt mit potenziell genotoxischen Stoffen kommen) an denselben behandelten Tieren die Reduktion von Tierversuchen 
Tab. 2 Verzehrsmengen von Lebensmitteln, basierend auf den Verwendungskategorien der

Materialien [22]

\begin{tabular}{|c|c|c|c|}
\hline Kategorie & $\begin{array}{l}\text { Lebensmittelkategorie, für wel- } \\
\text { che das Lebensmittelkontaktma- } \\
\text { terial vorgesehen ist, das die zu } \\
\text { evaluierende Substanz enthält }\end{array}$ & $\begin{array}{l}\text { Relevante Bevöl- } \\
\text { kerungsgruppe }\end{array}$ & $\begin{array}{l}\text { Verzehrsmenge, die } \\
\text { für die Expositionsab- } \\
\text { schätzung zu berück- } \\
\text { sichtigen ist (g/kg KG } \\
\text { pro Tag) }\end{array}$ \\
\hline 1 & $\begin{array}{l}\text { Wasser und Inhalte von Säuglings- } \\
\text { flaschen wie rekonstituierte Milch }\end{array}$ & Säuglinge & 150 \\
\hline 2 & $\begin{array}{l}\text { Milch, Milchprodukte und andere } \\
\text { nichtalkoholische Getränke (z. B. } \\
\text { Frucht- und Gemüsesäfte) }\end{array}$ & Kleinkinder & 80 \\
\hline 3 & $\begin{array}{l}\text { Feste Nahrung, die speziell für } \\
\text { Säuglinge und Kleinkinder be- } \\
\text { stimmt ist }\end{array}$ & Kleinkinder & 50 \\
\hline 4 & $\begin{array}{l}\text { Lebensmittel, die nicht durch die } \\
\text { Kategorien 1, } 2 \text { und } 3 \text { abgedeckt } \\
\text { sind }\end{array}$ & Kleinkinder & 20 \\
\hline
\end{tabular}

und gleichzeitig eine verbesserte Datenlage zur Genotoxizität im Säugetierkörper [28].

In den letzten Jahren hat besonders die Belastung von Lebensmitteln mit endokrin aktiven Substanzen wie Bisphenol A [12] oder bestimmten Phthalaten [18] zu einer Kontroverse bezüglich ihrer gesundheitlichen Bewertung und daraus folgenden vorbeugenden Maßnahmen zum Gesundheitsschutz bzw. Verwendungsverboten geführt (Bisphenol A: Durchführungsverordnung (EU) Nr. 321/2011, Verordnung (EU) Nr. 2016/2235; Diethylhexylphthalat: ECHA) [9]. Der Besorgnis, dass derartige Substanzen schon in geringen Dosen adverse Effekte induzieren oder zu deren Entstehung beitragen könnten [29], hat die EFSA [22] in ihrem Diskussionspapier zu Lebensmittelkontaktmaterialien Rechnung getragen, indem sie auch in der unteren Expositionsstufe Informationen zu endokrinen Effekten verlangt. Bei höheren Expositionen ( $a b \quad 1,5 \mu \mathrm{g}$ / kgKG pro Tag), die tierexperimentelle Daten erfordern, wird von der EFSA [22] bei Hinweisen auf endokrine Aktivität oder andere Wirkungen, für die die vorund nachgeburtlichen Lebensphasen besonders sensitiv sind, die Durchführung der (sub)chronischen Studien mit einer perinatalen Exposition vorgesehen. Eine solche Studie ist bereits für Bisphenol A durchgeführt [30] und von der EFSA [12] bewertet worden. Alternativ wird von der EFSA für Substanzen mit einer möglichen endokrinen und/oder entwicklungstoxischen Wirkung auf das Nerven- bzw. Immunsystem die erweiterte Eingenerationsstudie (EOGRTS: OECD TG 443) empfohlen. Toxikologische Informationen aus Screeningstudien zur Reproduktionstoxizität oder zu endokrinen Effekten können eine gezielte Durchführung der EOGRTS ermöglichen und helfen, die einzusetzenden Tierzahlen zu reduzieren [31]. Gleichzeitig ermöglichen die Daten aus einer EOGRTS auch die Bewertung der reproduktionstoxischen Eigenschaften. Deshalb kann die EOGRTS von der EFSA als Alternative zu einer Mehrgenerationsstudie (OECD TG 416) akzeptiert werden, was wiederum einer Reduktion von Tierzahlen zugutekommt. Für die Prüfung auf entwicklungsbedingte Wirkungen auf Verhalten und Neurotoxizität steht eine gezielte Richtlinie zur Verfügung (OECD 426). Dagegen gibt es für die Testung auf Toleranzentwicklung und allergische Reaktionen keine international anerkannten Verfahren, was die Substanzbewertung bei Vorliegen von Studien mit unterschiedlichen Testprotokollen - z. B. im Fall von Bisphenol A [32, 33] - erschwert.

Die Kunststoff-Verordnung (EU) 10/2011 fordert, dass nicht nur die beantragte Substanz, sondern auch migrierende Verunreinigungen und Abbauoder Reaktionsprodukte als unbeabsichtigt eingebrachte Stoffe (NIAS) gemäß international anerkannter wissenschaftlicher Grundsätze bewertet werden. Für NIASs liegen häufig keine oder nur unzu- reichende toxikologische Informationen vor. In diesen Fällen sind In-silico-Methoden hilfreich, um eine Einschätzung des gesundheitlichen Risikos der NIASs abzugeben. Hierfür eignet sich der Vergleich mit strukturell ähnlichen Substanzen („category approach“, „read across“) [34], wenn die vorhandenen toxikokinetischen und toxikologischen Daten die Übertragung von der/den Vergleichssubstanz/en wissenschaftlich gerechtfertigt erscheinen lassen. Als weitere international akzeptierte In-silico-Methode kann das bereits erwähnte TTC-Konzept und die Einordnung in Cramer-Klassen mit entsprechenden Expositionsschwellenwerten Anwendung finden [35, 36], wenn die chemische Struktur der NIASs hinreichend bekannt ist und keine Ausschlusskriterien für die Anwendung des TTC-Konzepts anzunehmen sind. Das BfR hat diese Methoden z. B. bei der Bewertung von Cyclo-di(Bisphenol-Amonoglycidylether) (CdB) angewandt [37]. Diese Substanz entsteht als NIAS bei der Herstellung von Epoxidharzen für Doseninnenbeschichtungen und migriert vor allem in fetthaltige Lebensmittel. Ein anderes Beispiel für NIASs sind Oligomere, die im Polymerisationsprozess von Kunststoffen oder bei deren Hydrolyse entstehen. Das BfR hat z. B. ein zyklisches Dimer aus Polyamid-Küchenutensilien, das besonders beim Kochen auf Lebensmittel übergehen kann, einer vorläufigen Bewertung unterzogen [38].

\section{Neue Technologien}

\section{Verwendung von Nanomaterialien}

Ein weitere Herausforderung in der Bewertung von Stoffen aus Materialien für den Lebensmittelkontakt stellen nanoskalige Stoffe dar [39]. Empfehlungen zu Teststrategien derartiger Stoffe in Lebensmitteln hat die EFSA bereits 2011 veröffentlicht [40]. Inzwischen sind verschiedene Substanzen in Nanoform zur Herstellung von Lebensmittelkontaktmaterialien von der EFSA bewertet worden $[41,42]$. Bei den für diesen Verwendungszweck bisher bewerteten Nanomaterialien wurde (aufgrund von Migrationsmodellierungen) davon ausgegangen, dass 
Tab. 3 Datenanforderungen gemäßEFSA „Note for Guidance“; in Klammern sind die Vorschläge der EFSA angegeben [22]

\begin{tabular}{|c|c|}
\hline $\begin{array}{l}\text { Bisher: Übergänge in Le- } \\
\text { bensmittel/-Simulanzien } \\
\text { (mg/kg) } \\
\text { EFSA-Vorschlag: Exposition } \\
\text { ( } \mathrm{gg} / \mathrm{kg} \mathrm{KG/Tag)}\end{array}$ & $\begin{array}{l}\text { Vorzulegende toxikologische Daten } \\
\text { (EFSA-Vorschlag) }\end{array}$ \\
\hline \multirow{3}{*}{$\begin{array}{l}<0,05 \mathrm{mg} / \mathrm{kg} \\
(<1,5 \text { bzw. }<30, \text { wenn } \\
\text { Cramer-Klasse I) }\end{array}$} & 3 bzw. $2^{\text {a }}$ Mutagenitätsstudien in vitro \\
\hline & (Verfügbare Informationen, z. B. ECHA-Datenbasis) \\
\hline & $\begin{array}{l}\text { (Falls endokrine, neuro- oder immuntoxische, akkumulierende, } \\
\text { nanoskalige Eigenschaften vorhanden, können mehr Daten } \\
\text { erforderlich werden.) }\end{array}$ \\
\hline \multirow{3}{*}{$\begin{array}{l}0,05-5 \\
(<80)\end{array}$} & Zusätzlich: \\
\hline & (Erweiterte) 90-Tage-Studie, oral \\
\hline & Daten zum Akkumulationspotenzial \\
\hline \multirow{4}{*}{$\begin{array}{l}>5-60 \\
(>80)\end{array}$} & Zusätzlich: \\
\hline & $\begin{array}{l}\text { Studien zu Absorption, Verteilung, Metabolismus und Aus- } \\
\text { scheidung }\end{array}$ \\
\hline & Studien zur Reproduktions- und Entwicklungstoxizität \\
\hline & (Erweiterte) Langzeitstudien (Toxizität und Kanzerogenität) \\
\hline
\end{tabular}

diese zu keiner relevanten Verbraucherexposition führen. Die Problematik der Bewertung von Nanomaterialien wurde von der EFSA [22] diskutiert und besteht einerseits in der Präzision der analytischen Methoden, um eine Freisetzung und Bioverfügbarkeit von nanoskaligen Stoffen ausschließen zu können, zum anderen im Fehlen eines Schwellenwerts, unterhalb dessen keine toxikologischen Bedenken für diese Stoffe bestehen. Die Bewertung erfordert daher eine fallspezifische Betrachtung, die vom Übergang der Nanomaterialien ins Lebensmittel sowie ihrem Verhalten im Körper, d. h. ihrer Bioverfügbarkeit in partikulärer oder löslicher Form, abhängig ist [43].

\section{Aktive und intelligente Materialien und Gegenstände}

Aktive Materialien und Gegenstände sind dazu bestimmt, die Haltbarkeit eines verpackten Lebensmittels zu verlängern oder dessen Zustand zu erhalten bzw. zu verbessern. Sie enthalten gezielt Bestandteile, die Stoffe an das verpackte Lebensmittel oder die das Lebensmittel umgebende Umwelt abgeben („Releaser“) oder diesen Stoffe entziehen („Absorber"). Die gezielte Abgabe von Stoffen muss dabei in Übereinstimmung mit den für Lebensmittelzusatzstoffe, Aromastof- fe und Enzyme geltenden Vorschriften stehen, d.h. es dürfen nur die für diese Zwecke zugelassenen Stoffe unter Einhaltung der lebensmittelrechtlich festgelegten Beschränkungen verwendet werden.

Unter intelligenten Materialien und Gegenständen werden Systeme verstanden, mit denen der Zustand eines verpackten Lebensmittels oder die das Lebensmittel umgebende Umwelt überwacht werden. Daskönnen beispielsweise Zeit-Temperatur-Indikatoren sein, die durch eine Farbveränderung Temperaturüberschreitungen in der Lieferkette oder den Ablauf von Haltbarkeitsfristen anzeigen.

Entsprechend der Verordnung (EG) 459/2009 zu aktiven und intelligenten Materialien und Gegenständen für den Lebensmittelkontakt sind ein Antragsverfahren mit Bewertung durch die EFSA und die Aufnahme in eine Gemeinschaftsliste für Substanzen vorgesehen, die in Releaser-Systemen verwendet werden sollen (z. B. zur Verstärkung der Wirkung der aktiven Substanz oder als Ausgangsstoff für die Bildung von aktiven Substanzen) oder die Bestandteile von Absorber-Systemen oder intelligenten Materialien und Gegenständen sind. Beispiele für Absorber-Systeme sind Saugeinlagen, die in Verpackungen für Frischfleisch oder -fisch verwendet werden, um austretende Flüssigkeit aufzunehmen, oder auch Absorber für gasförmige Stoffe wie Sauerstoff und Ethylen.

Die EFSA hat eine Leitlinie zur Einreichung eines Antrags auf Sicherheitsbewertung von Substanzen in aktiven und intelligenten Materialien und Gegenständen erarbeitet [44], die den Bewertungsgrundsätzen des EFSA Note for Guidance folgt.

\section{Wiederverwendung recyclierter Materialien im Lebensmittel- kontakt}

Die Anforderungen an die gesundheitliche Unbedenklichkeit von Stoffübergängen stellen für recyclierte Materialien eine große Herausforderung dar. Die Verwendung recyclierter Kunststoffe ist auf europäischer Ebene durch die Verordnung (EG) 282/2008 geregelt. Als Ausgangsmaterial dürfen nur Kunststoffe zum Einsatz kommen, die den Anforderungen der Kunststoff-Verordnung (EU) 10/2011 entsprechen. Die Verordnung sieht eine Zulassung der Recyclingverfahren vor. Für das PETRecycling hat die EFSA Grundsätze für die Sicherheitsbewertung [45] und Leitlinien für die Antragstellung erarbeitet [46]. Im Zulassungsverfahren werden die Risiken durch den möglichen Übergang von Kontaminanten aus

- der vorherigen Verwendung einschließlich dem möglichen Missbrauch,

- Ausgangsmaterialien, die in ihrer Zusammensetzung nicht den Anforderungen für den Lebensmittelkontakt entsprechen,

- der Verwendung von Chemikalien im Recyclingprozess,

- dem möglichen Abbau des Kunststoffs beim Recycling

bewertet. Dafür ist ein Challenge-Test vorzulegen, in welchem dem Ausgangsmaterial für das Recycling Surrogatkontaminanten zugesetzt werden und die Effektivität des Verfahrens zur Entfernung dieser Stoffe geprüft wird [47].

Für recyclierte Papiere und Kartons, die eine breite Anwendung im Lebens- 
Infobox 1 Relevante Kriterien für die Bewertung von Kunststoffen (EFSA) und anderen Materialien (z. B. BfR, RIVM, ANSES, DTU)

- Identität einschl. Verunreinigungen (Einzelsubstanz, definierte/nicht definierte Mischung, polymere Additive)

- physikalisch-chemische Eigenschaften, Reaktionsprodukte

- technologische Funktion, Einsatzmenge, Verarbeitungstemperatur, Kontaktbedingungen

- bestehende Zulassungen

- Daten zum Übergang auf Lebensmittel im ungünstigsten vorhersehbaren Fall einschl. von Analysenmethoden (beantragte Substanz, ggf. Reaktions- und Abbauprodukte, ggf. Oligomere bis 1000 $\mathrm{Da})$

- Daten zum Restgehalt im Material einschl. von Analysenmethoden

- spezifische Informationen zu antimikrobiell wirksamen Stoffen

- toxikologische Daten in Abhängigkeit vom Übergang auf Lebensmittel

mittelkontakt finden, existieren keine vergleichbaren Vorschriften. Der Übergang von Stoffen, die aus der Wiederverwendung von Papierfasern stammen, wird im Einzelfall bewertet. Für einige bekannte Substanzen, die über das Papier-Recycling eingetragen werden können, sind in der BfR-Empfehlung XXXVI zu Papieren, Kartons und Pappen für den Lebensmittelkontakt [48] Richtwerte für die Beurteilung des Übergangs auf Lebensmittel aufgeführt. Eine besondere Herausforderung stellt die Bewertung von Mineralölbestandteilen dar, die aus den im Zeitungsdruck verwendeten Druckfarben in den Papierkreislauf gelangen und im Recyclingprozess derzeit nicht vollständig entfernt werden können. Toxikologische Daten zu den Substanzgemischen, deren Zusammensetzung variabel und nicht ausreichend charakterisierbar ist, stehen nicht zur Verfügung.

\section{Offene Fragen}

Erhebliche Auswirkungen auf das Risikomanagement unter der Verordnung (EU) 10/2011 können sich aus dem Vorschlag der EFSA [22] ergeben, anstelle der Höhe des Übergangs auf Lebensmittel eine abgestufte Abschätzung der Exposition zum
Ausgangspunkt für die gesundheitliche Bewertung zu machen. Die EU-Kommission behält sich vor, vor der Anwendung des EFSA-Vorschlags diese Auswirkungen zu prüfen. Dies ist bislang nicht erfolgt.

Das europäische Regelungswerk zu Materialien für den Lebensmittelkontakt nimmt über allgemeine Anforderungen hinaus vor allem auf Kunststoffe Bezug. Deshalb hat die EFSA im Rahmen der Scientific Cooperation eine Arbeitsgruppe etabliert, in der Vertreter aus den Lebensmittelsicherheitsbehörden der Mitgliedstaaten Informationen zu den vorhandenen nationalen Bewertungen und den angewandten Bewertungsgrundsätzen zusammengetragen haben [4]. Diese Kooperation wird im EFSANetzwerk zu Lebensmittelbedarfsgegenständen weitergeführt [49].

Die Bewertungen der EFSA zu Bestandteilen von aktiven und intelligenten Materialien beziehen sich zumeist auf Systeme, deren Effekt auf dem $\mathrm{Zu}-$ sammenwirken mehrerer Bestandteile beruht, und schließen auch entstehende Reaktionsprodukte ein. Entsprechend dem Regelungsansatz der Verordnung (EG) 459/2009 sollen die zulässigen Stoffe in der vorgesehenen Gemeinschaftsliste jedoch einzeln aufgeführt und dann auch in anderen als durch die EFSA-Bewertung berücksichtigten Kombinationen verwendet werden.

Die Bewertungen der EFSA zur Aufnahme von Substanzen in die Positivliste der Verordnung (EU) 10/2011 können sich nur auf die Informationen zum Übergang von Stoffen auf Lebensmittel beziehen, die im Antragsverfahren eingehen. Sie sind auf ein konkretes, oft auch speziell für die Antragstellung hergestelltes Material bezogen. Die Verordnung lässt eine allgemeine Verwendung der gelisteten Stoffe zu. NIASs, die bei der möglichen Verwendung unter anderen Bedingungen, z. B. in anderen Polymeren oder bei Änderungen im Herstellungsverfahren, entstehen, sind jedoch bei der Bewertung nicht absehbar. Die analytischen Prüfungen und die Beurteilung der gesundheitlichen Unbedenklichkeit verbleiben daher in der Verantwortung des Herstellers oder Inverkehrbringers, stellen aber auch an die Lebensmittelüber- wachung in den Mitgliedsstaaten der EU hohe Anforderungen.

Wie in anderen Regelungsbereichen bestehen auch für Materialien für den Lebensmittelkontakt offene Fragen hinsichtlich der Bewertung und Regulation von Substanzgemischen und endokrin aktiven Substanzen. Hier ist eine grundsätzliche Klärung durch die Europäische Kommission notwendig. Soweit die ungelösten Fragen in der Substanzbewertung nicht durch bessere Konzepte oder zusätzliche Daten beantwortet werden, können zukünftig Unsicherheitsanalysen ein weiteres Element in der Substanz-/Gemischbewertung darstellen [50], um den Risikomanagern für die Regulation von Substanzen eine möglichst transparente wissenschaftliche Bewertungsgrundlage zur Verfügung zu stellen.

\section{Korrespondenzadresse}

\section{Dr. K. Pfaff}

Bundesinstitut für Risikobewertung

Max-Dohrn-Str. 8-10, 10589 Berlin,

Deutschland

Karla.Pfaff@bfr.bund.de

\section{Einhaltung ethischer Richtlinien}

Interessenkonflikt. K. Pfaff, D. Wölfle und A. Luch geben an, dass kein Interessenkonflikt besteht.

Dieser Beitrag beinhaltet keine von den Autoren durchgeführten Studien an Menschen oder Tieren.

Open Access. Dieser Artikel wird unter der Creative Commons Namensnennung 4.0 International Lizenz (http://creativecommons.org/licenses/by/4.0/deed. de) veröffentlicht, welche die Nutzung, Vervielfältigung, Bearbeitung, Verbreitung und Wiedergabe in jeglichem Medium und Format erlaubt, sofern Sie den/die ursprünglichen Autor(en) und die Quelle ordnungsgemäßnennen, einen Link zur Creative Commons Lizenz beifügen und angeben, ob Änderungen vorgenommen wurden.

\section{Literatur}

1. European Parliament (2016) Implementation of the Food Contact Materials Regulation. European Parliament resolution of 6 October 2016 on the implementation of the Food Contact Materials Regulation (EC) No 1935/2004 (2015/2259(INI)). http://www.europarl.europa.eu/sides/getDoc. do?pubRef=-//EP//NONSGML+TA+P8-TA-20160384+0+DOC+PDF+V0//EN. Zugegriffen: 09 Feb 2017 
2. Wölfle D, Pfaff K (2010) Sicherheitsbewertung von Materialien im Kontakt mit Lebensmitteln. Bundesgesundheitsblatt Gesundheitsforschung Gesundheitsschutz 53:561-566

3. Geueke B, Wagner CC, Muncke J (2014) Food contact substances and chemicals of concern: a comparison of inventories. Food Addit Contam 31(8):1438-1450

4. EFSA (2012) External scientific report. Report of ESCO WG on non-plastic food contact materials. EFSA Support Publ 2012:EN-139

5. BfR (2017) Datenbank BfR-Empfehlungen zu Materialien für den Lebensmittelkontakt. https://bfr. ble.de/kse/faces/DBEmpfehlung.jsp. Zugegriffen: 09 Feb 2017

6. BfR (2013) Primäre aromatische Amine aus bedruckten Lebensmittelbedarfsgegenständen wie Servietten oder Bäckertüten. Stellungnahme Nr. 021/2014 des BfR vom 24. Juli 2013. http://www.bfr.bund.de/cm/343/ primaere-aromatische-amine-aus-bedrucktenlebensmittelbedarfsgegenstaenden-wieservietten-oder-baeckertueten.pdf. Zugegriffen: 09 Feb 2017

7. Trier $X$, Okholm B, Foverskov A, Binderup $M-L$, Petersen JH (2010) Primary aromatic amines (PAAs) in black nylon and other food-contact materials, 2004-2009. Food Addit Contam 27(9):1325-1335

8. BfR (2013) Fragen und Antworten zu PhthalatWeichmachern. FAQ des BfR und des Umweltbundesamtes (UBA) vom 7. Mai 2013. http://www. bfr.bund.de/cm/343/fragen-und-antworten-zuphthalat-weichmachern.pdf. Zugegriffen: 09 Feb 2017

9. ECHA (2014) Agreement of the member state committee on the identification of Bis(2-ethylhexyl) phthalate (DEHP) as a substance of very high concern. https://echa.europa.eu/documents/ 10162/ad79f939-cd8e-4eab-8fee-59288fecbf00. Zugegriffen: 09Feb 2017

10. Pabel U, Buhrke T, Abraham K et al (2017) Persistente organische Kontaminanten in Lebensmitteln. Manuskript zur Veröffentlichung in Bundesgesundheitsbl Gesundheitsforsch Gesundheitsschutz

11. ECHA (2015) Committee for Risk Assessment (RAC). Opinion on an Annex XV dossier proposing restrictions on Perfluorooctanoic acid (PFOA), its salts and PFOA-related substances. https://echa. europa.eu/documents/10162/3d13de3a-de0d49ae-bfbd-749aea884966. Zugegriffen: 09 Feb 2017

12. EFSA (2015) Scientific opinion on the risks to public health related to the presence of bisphenol $A$ (BPA) in foodstuffs: executive summary. EFSA J 13(1):3978

13. ECHA (2016) Member state committee support document for identification of 4,4'-Isopropylidenediphenol (Bisphenol A) as a substance of very high concern because of its toxic for reproduction (Article 57 c) properties. https://echa.europa.eu/ documents/10162/b10d6a00-8e47-9b14-4f61c779a8dc8450. Zugegriffen:09 Feb 2017

14. BfR (2015) Fragen und Antworten zu Aluminium in Lebensmitteln und verbrauchernahen Produkten. Aktualisierte FAQ des BfR vom 20. Februar 2015. http://www.bfr.bund.de/cm/343/fragen-undantworten-zu-aluminium-in-lebensmittelnund-verbrauchernahen-produkten.pdf. Zugegriffen:09 Feb 2017

15. BfR (2011) 7. Sitzung der BfR-Kommission für Bedarfsgegenstände. Protokoll der Sitzung vom 14. April 2011. http://www.bfr.bund.de/ cm/343/7_sitzung_der_bfr_kommission_fuer bedarfsgegenstaende.pdf. Zugegriffen: 09 Feb 2017

16. EFSA (2012) Scientific opinion on mineral oil hydrocarbons in food. EFSA J 10(6):2704

17. van Bossuyt $M$, van Hoeck $E$, Vanhaecke $T$, Rogiers V, Mertens B (2016) Printed paper and board food contact materials as a potential source of food contamination. Regul Toxicol Pharmacol 81:10-19

18. Howdeshell KL, Rider CV, Wilson VS, Furr JR, Lambright CR, Gray LE Jr (2015) Dose addition models based on biologically relevant reductions in fetal testosterone accurately predict postnata reproductive tract alterations by a phthalate mixture in rats. Toxicol Sci 148(2):488-502

19. Fromme H, Wöckner M, Roscher E, Völkel W (2016) ADONA and perfluoroalkylated substances in plasma samples of German blood donors living in South Germany. Int J Hyg Environ Health. doi:10. 1016/j.ijheh.2016.12.014

20. Barp L, Biedermann M, Grob K et al (2017) Accumulation of mineral oil saturated hydrocarbons (MOSH) in female Fischer 344 rats: comparison with human data and consequences for risk assessment. Sci Total Environ 575:1263-1278

21. Grob K, Spinner C, Brunner M, Etter R (2010) The migration from the internal coatings of food cans; summary of the findings and call for more effective regulation of polymers in contact with foods: a review. Food Addit Contam 16(7):579-590

22. EFSA (2016) Recent developments in the risk assessment of chemicals in food and their potential impact on the safety assessment of substances used in food contact materials. EFSA J 14(1):4357

23. EFSA (2008) Note for Guidance for the preparation of an application for the safety assessment of a substance to be used in plastic food contact materials. http://onlinelibrary.wiley.com/doi/10. 2903/j.efsa.2008.21r/epdf. Zugegriffen: 09 Feb 2017

24. VKM (2009) Norwegian Scientific Committee for Food Safety Evaluation of the EU exposure model for migration from food contact materials (FCM). http://www.vkm.no/dav/2a7d1 ce442.pdf Zugegriffen:09Feb 2017

25. EFSA (2011) The EFSA Comprehensive European Food Consumption Database. http:// www.efsa.europa.eu/en/food-consumption/ comprehensive-database. Zugegriffen: 09 Feb 2017

26. EFSA (2011) Scientific opinion on genotoxicity testing strategies applicable to food and feed safetyassessment. EFSA J 9(9):2379

27. Bolognesi C, Castoldi AF, Crebelli R et al (in review) Genotoxicity testing approaches for the safety assessment of substances used in food contact materials prior to their authorisation in the European Union. Environ Mol Mutagen

28. Frötschl R (2015) Experiences with the in vivo and in vitro comet assay in regulatory testing. Mutagenesis 30(1):51-57

29. Gore AC, Chappell VA, Fenton SE et al (2015) Executive summary to EDC-2: the Endocrine Society's second scientific statement on endocrinedisrupting chemicals. Endocr Rev 36(6):593-602

30. Delclos KB, Camacho L, Lewis SM et al (2014) Toxicity evaluation of bisphenol $A$ administered by gavage to Sprague Dawley rats from gestation day 6 through postnatal day 90. Toxicol Sci 139(1):174-197

31. Moore NP, Beekhuijzen M, Boogaard PJ et al (2016) Guidance on the selection of cohorts for the extended one-generation reproduction toxicity study (OECD test guideline 443). Regul Toxicol Pharmacol 80:32-40
32. Hessel EV, Ezendam J, van Broekhuizen FA (2016) Assessment of recent developmental immunotoxicity studies with bisphenol $A$ in the context of the 2015 EFSA t-TDI. Reprod Toxicol 65:448-456

33. EFSA (2016) A statement on the developmental immunotoxicity of bisphenol A (BPA): answer to the question from the Dutch Ministry of Health Welfare and Sport. EFSA J 14(10):4580

34. ECHA (2015) Read-Across Assessment Framework (RAAF). https://echa.europa.eu/documents/ 10162/13628/raaf_en.pdf. Zugegriffen: 09 Feb 2017

35. EFSA(2012)Scientific opinion on exploring options for providing advice about possible human health risks based on the concept of Threshold of Toxicological Concern (TTC). EFSA J 10(7):2750

36. Partosch F, Mielke H, Stahlmann R (2015) Internal threshold of toxicological concern values: enabling route-to-route extrapolation. Arch Toxicol 89(6):941-948

37. BfR (2016) Epoxidharz-Beschichtungen von Konservendosen: Stoffübergänge in ölhaltige Lebensmittel sind möglich. Stellungnahme 022/2016 des BfR vom 15. April 2016. http://www.bfr. bund.de/cm/343/epoxidharz-beschichtungenvon-konservendosen-stoffuebergaenge-inoelhaltige-lebensmittel-sind-moeglich.pdf. Zugegriffen:09 Feb 2017

38. BfR (2012) 10. Sitzung der BfR-Kommission für Bedarfsgegenstände. Protokoll vom 29. November 2012. http://www.bfr.bund. $\mathrm{de} / \mathrm{cm} / 343 / 10$-sitzung-der-bfr-kommissionfuer-bedarfsgegenstaende.pdf. Zugegriffen: 09.02.2017

39. Peters RJB, Bouwmeester H, Gottardo S et al (2016) Nanomaterials for products and application in agriculture, feed and food. Trends Food Sci Technol 54:155-164

40. EFSA (2011) Guidance on the riskassessment of the application of nanoscience and nanotechnologies in the food and feed chain. EFSA J 9(5):2140

41. EFSA (2012) Scientific opinion on the safety evaluation of the substance, titanium nitride, nanoparticles, for use in food contact materials. EFSA J 10(3):264

42. EFSA (2016) Safety assessment of the substance zinc oxide, nanoparticles, for use in food contact materials. EFSA J 14(3):4408

43. Jokar M, Pedersen GA, Loeschner K (2016) Six open questions about the migration of engineered nano-objects from polymer-based food-contact materials: a review. Food Addit Contam Part A Chem Anal Control Expo Risk Assess 22:1-17

44. EFSA (2009) Opinion of the Panel on food contact materials, enzymes, flavourings and processing aids (CEF) Guidelines on submission of a dossier for safety evaluation by the EFSA of active or intelligent substances present in active and intelligent materials and articles intended to come into contact with food. EFSA J 1208:1-11

45. EFSA (2011) Scientific opinion on the criteria to be used for safety evaluation of a mechanical recycling process to produce recycled PET intended to be used for manufacture of materials and articles in contact with food. EFSA J9(7):2184

46. EFSA (2008) Opinion of the Scientific Panel on food additives, flavourings, processing aids and materials in contact with food (AFC) on Guidelines on submission of a dossier for safety evaluation by the EFSA of a recycling process to produce recycled plastics intended to be used for manufacture of materials and articles in contact with food. EFSA J 717:1-12 


\section{Leitthema}

47. Barthélémy $E$, Spyropoulos $D$, Milana M-R et al (2014) Safety evaluation of mechanical recycling processes used to produce polyethylene terephthalate (PET) intended for food contact applications. Food Addit Contam Part A Chem Anal Control Expo Risk Assess 31(3):490-497

48. BfR (2016) BfR-Empfehlung XXXVI. Papiere, Kartons und Pappen für den Lebensmittelkontakt. Stand vom 01.07.2016. https:// bfr.ble.de/kse/faces/resources/pdf/360.pdf; jsessionid=A98AF6ACA121C2312A66CC4BA7B D175F.Zugegriffen:09 Feb 2017

49. EFSA Scientific Network on Food Contact Materials (FCM) https://www.efsa.europa.eu/en/foodingredients-and-packaging/networks. Zugegriffen:09.02.2017

50. EFSA (2016) Outcome of a public consultation on the draft guidance document of the EFSA Scientific Committee on Uncertainty in EFSA Scientific Assessment. EFSA Support Publ 2016:EN-1011 